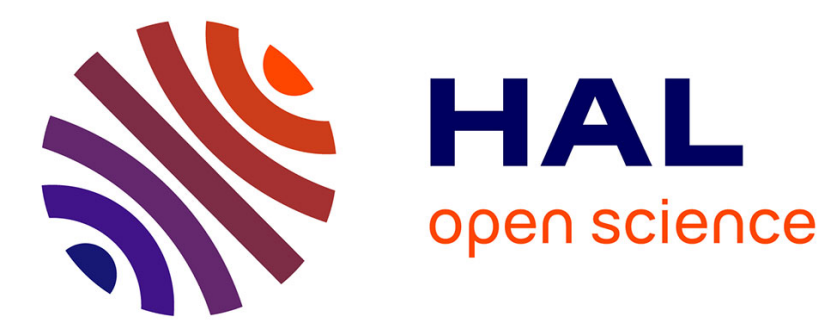

\title{
Une étude de la répartition du pouvoir confessionnel au Liban
}

Mostapha Diss, Abdallah Zouache

\section{To cite this version:}

Mostapha Diss, Abdallah Zouache. Une étude de la répartition du pouvoir confessionnel au Liban. 2014. halshs-01090114v2

\section{HAL Id: halshs-01090114 \\ https://shs.hal.science/halshs-01090114v2}

Preprint submitted on 2 Feb 2015

HAL is a multi-disciplinary open access archive for the deposit and dissemination of scientific research documents, whether they are published or not. The documents may come from teaching and research institutions in France or abroad, or from public or private research centers.
L'archive ouverte pluridisciplinaire HAL, est destinée au dépôt et à la diffusion de documents scientifiques de niveau recherche, publiés ou non, émanant des établissements d'enseignement et de recherche français ou étrangers, des laboratoires publics ou privés. 
Une étude de la répartition du pouvoir confessionnel au Liban 


\section{GATE Groupe d'Analyse et de Théorie Économique Lyon-St Étienne}

93, chemin des Mouilles 69130 Ecully - France

Tel. +33 (0)4 72866060

Fax $+33(0) 472866090$

6, rue Basse des Rives 42023 Saint-Etienne cedex 02 - France

Tel. +33(0)4 77421960

Fax. +33 (0)4 77421950

Messagerie électronique / Email : gate@gate.cnrs.fr

Téléchargement / Download : http://www.gate.cnrs.fr - Publications / Working Papers 


\title{
Une étude de la répartition du pouvoir confessionnel au Liban
}

\author{
Mostapha Diss* $\quad$ Abdallah Zouache ${ }^{\dagger}$
}

29 janvier 2015

\begin{abstract}
Résumé
La composition du parlement libanais est basée sur un compromis entre les différentes confessions religieuses de ce pays, souvent considéré comme seule démocratie parlementaire de l'Orient arabe. À partir des outils de la théorie des jeux coopératifs, cet article analyse le pouvoir des différentes confessions dans le parlement libanais. Nous étudions la différence entre le pouvoir "apparent" et le pouvoir "réel" de chaque confession prenant part au processus de décision. Cet article analyse aussi l'impact des dispositions de la nouvelle réforme électorale sur le pouvoir de chaque confession. Nous montrerons que le compromis confessionnel au Liban est loin d'être parfait.
\end{abstract}

MOTS-CLÉS : Liban, pouvoir de vote, Banzhaf, Shapley-Shubik, religion.

\begin{abstract}
The composition of the Lebanese Parliament is based on the allocation of a specific number of seats to each of the various minority groups (confessions) in this country, which is considered to be the only parliamentary democracy in Arab Middle East. Using tools from cooperative game theory, this paper analyzes the power of different confessions in the Lebanese parliament. We study the difference between the "apparent" power and the "real" power of each minority group involved in the decision process. This paper also analyzes the impact of the new electoral reform on the power of each confession. We will show that the confessional compromise in Lebanon is far from being flawless.
\end{abstract}

KEYWORDS: Lebanon, voting power, Banzhaf, Shapley-Shubik, religion.

ClassifiCATION JEL : D71, D72

\section{Introduction}

La principale spécificité du Liban concerne la nature confessionnelle de son système politique caractérisé par une diversité des groupes politiques confessionnels qui acceptent le principe des élections démocratiques. En ce sens, ce système tend à promouvoir un certain libéralisme politique caractérisé par une liberté religieuse, une expression assez libre et une liberté d'association (Makdisi et Sadaka, [35]). Il est possible d'envisager que ce système politique a constitué une protection à la vague de protestations qui ont fait suite à ce que l'on a appelé le printemps arabe (Owen, [41]). En parallèle, la dimension confessionnelle de la vie politique libanaise est également estimée comme un facteur favorisant la formation de groupes d'intérêts particuliers, en luttes constantes pour le pouvoir et n'hésitant pas à utiliser le clientélisme, le népotisme voire la corruption comme instruments d'atteinte du pouvoir.

\footnotetext{
*Auteur Correspondant : Université de Lyon, Lyon, F-69007, France; CNRS, GATE Lyon Saint-Etienne, Ecully, F-69130, France ; Université Jean Monnet, Saint-Etienne, F-42000, France. Courriel : diss@ gate.cnrs.fr.

${ }^{\dagger}$ Sciences Po Lille, 84, rue de Trévise 59000 Lille, France. Courriel : abdallah.zouache@ sciencespo-lille.eu.
} 
L'un des enjeux majeurs du système confessionnel au Liban est le découpage de chaque circonscription électorale et la répartition des sièges parlementaires entre les communautés. Plus précisément, les sièges du Parlement sont divisés à part égale entre chrétiens et musulmans et, à l'intérieur, ces sièges sont de nouveau subdivisés entre toutes les divisions confessionnelles. Il nous semble que ce caractère particulier fait du Liban un cas propice à étudier par la théorie des jeux coopératifs qui offre des outils permettant d'identifier les bénéficiaires de ce système confessionnel. En effet, l'objectif principal de cet article est d'étudier la répartition du pouvoir entre les différentes confessions au sein du parlement en faisant appel aux indices de pouvoir de Banzhaf [4] et de Shapley-Shubik [43]. Dans cette perspective, notre article s'efforce de distinguer le pouvoir "réel" et le pouvoir "apparent" de chaque confession en prenant en compte la répartition actuelle des sièges entre les confessions ainsi que la prochaine configuration qui fera suite à une nouvelle réforme qui entrera en application lors des prochaines élections législatives. Autrement dit, nous faisons appel à l'indice de Banzhaf et à celui de Shapley-Shubik pour examiner dans quelle mesure le pouvoir distribué correspond au pouvoir effectif de chaque confession. Nous allons également analyser l'effet de la nouvelle réforme sur la distribution du pouvoir de chaque confession.

Nous contribuons, à travers cet article, à la littérature consacrée aux indices de pouvoir qui s'est considérablement étendue durant les dernières décennies. Les deux indices que nous utilisons dans notre application émergent de cette littérature dans la mesure où les premiers débats se sont essentiellement concentrés sur l'analyse et la comparaison des deux indices. Au niveau théorique, à notre connaissance, ces indices n'avaient aucune fondation axiomatique avant Dubey [19] et Dubey et Shapley [20]. Depuis, ils ont été caractérisés de multiples manières par différents auteurs. De nombreux travaux s'inscrivent dans cette perspective, tels que Barua et al [9], Laruelle et Valenciano [30], Nowak et Tradzik [36], Owen [38, 39], Straffin [44]. Par ailleurs, les travaux de Banzhaf [4] et Shapley-Shubik [43] ainsi que ceux de Penrose [42] et Coleman [14] ont été à l'origine de l'émergence de nouveaux indices dont la construction est, pour la plupart, inspirée des indices de Shapley-Shubik ou de Banzhaf. A titre d'exemple, nous pouvons citer l'indice de Colomer-Martinez (Colomer [15] et Colomer et Martinez [16]), Curiel [17], Deegan-Packel [18], Holler-Packel [27], Johnston [28], Owen [37, 40]. Au niveau empirique, l'essor de ces indices a été à l'origine de l'émergence de nombreuses applications. Straffin [44, 45] offre une comparaison et des applications intéressantes des indices de Shapley-Shubik et Banzhaf. Notons qu'une part importante de travaux de recherche a été consacrée à l'application des indices de pouvoir aux instances de l'Union Européenne en étudiant les capacités d'influence des Etats membres selon plusieurs configurations. Sachant que la littérature dans ce domaine est devenue considérable, nous renvoyons le lecteur aux travaux de Andjiga et al [2], Bobay [12, 13], Felsenthal et Machover [22, 23], Garrett et Tsebelis [24], Herne et Nurmi [25], Laruelle et Widgrén [31]. Des références plus récentes sont celles de Alonso-Meijide et al [1], Barthélémy et Martin [7, 8], Barthélémy et al [5, 6], Bisson et al [11], Kauppi et Widgrén [29] ou encore l'ouvrage de Holler et Nurmi [26] qui proposent des applications des indices de pouvoir.

L'article est organisé comme suit. La section 2 discute le système confessionnel libanais. La section 3 présente le cadre d'analyse, en introduisant un rappel du fonctionnement des indices de Banzhaf et Shapley-Shubik. Cette section discute aussi les résultats de notre application. Les extensions possibles ainsi que les limites de notre article seront évoquées en conclusion. 


\section{La mosaïque confessionnelle libanaise}

\subsection{Le contexte}

Bien que la constitution de l'Etat Libanais garantisse des droits égaux pour tous les citoyens, l'article 95 de la constitution de l'indépendance (1943) annonce que les communautés religieuses seront représentées de façon équitable dans l'emploi public et les postes ministériels ${ }^{1}$. Le principe de représentation équitable ne fait dès lors pas l'objet d'un texte écrit mais est le résultat d'un accord informel entre les leaders politiques. Cet accord stipule que la présidence de la république est dévolue à un chrétien maronite, le président du parlement doit être issu de la communauté chiite, et le poste de premier ministre est réservé à un musulman sunnite. Ceci s'accompagne d'une distribution sur le mode confessionnel des postes de haut fonctionnaire; notamment les postes dans les cabinets ministériels devant être distribués entre les six principales communautés religieuses du pays, tout en respectant plus globalement le principe d'équité entre les musulmans et les chrétiens.

Néanmoins, après l'indépendance, les sièges parlementaires sont à une faible majorité (55\%) attribués à la communauté chrétienne. De plus, le président est doté de pouvoirs exécutifs non négligeables : il préside le conseil des ministres, nomme le premier ministre et les membres du cabinet après consultation. De telles prérogatives présidentielles font dire à Makdisi et Sadaka [35] que la communauté maronite émergea comme la communauté religieuse la plus influente de 1943 à 1975 ; d'autant plus influente que ce positionnement politique lui permettait également d'avoir un poids économique plus important du fait notamment de la distribution des postes de gestion des administrations et des entreprises publiques. D'après ces auteurs, ce déséquilibre confessionnel rendait l'Etat libanais faible face à des chocs extérieurs, tels que la guerre de $1967^{2}$ qui a été suivie d'un afflux massif de réfugiés palestiniens. Une telle faiblesse expliquerait ainsi la guerre civile que le Liban a connu de 1975 à 1989.

L'accord de Taëf ${ }^{3}$ marque la fin de la guerre civile et institue le fonctionnement du système confessionnel actuel. L'accord conserve l'esprit d'un fonctionnement du système politique libanais sur une base confessionnelle. L'objectif est néanmoins de rééquilibrer ce système entre les deux principales communautés religieuses, les musulmans et les chrétiens. En particulier, la dimension présidentielle du régime est affaiblie. Le pouvoir du premier ministre, musulman sunnite, se trouve renforcé, ainsi que celui du président du parlement, chiite. Ainsi, la nouvelle constitution stipule que la nomination du premier ministre doit se faire en consultation avec les membres du parlement. La durée du mandat du président de la chambre passe de 1 an à 4 ans. De plus, l'accord spécifie une représentation égalitaire de la représentation des communautés musulmanes et chrétiennes. D'après certains commentateurs (Berthelot, [10]), cet accord a renforcé les sunnites, maintenus à la présidence d'un conseil doté de pouvoirs élargis. Le Hezbollah a été compensé par l'octroi "officiel" de la force militaire. La réduction du poids des chrétiens s'expliquerait par l'affaiblissement des prérogatives du président de la république. Néanmoins, ils conservent une surreprésentation au parlement avec 50\% des sièges en dépit de la baisse de leur poids démographique. Makdisi et Sadaka [35], quant à eux, considèrent que l'accord de Taëf a essentiellement institutionalisé une règle de fonctionnement politique qui persiste aujourd'hui sous le régime d'une troïka composée du président de la république, du président du parlement, et du premier ministre. Ceci nous amène à une description détaillée de la configuration du parlement en fonction du nombre de sièges attribués à chaque confession.

\footnotetext{
${ }^{1}$ Cette section s'inspire des contributions de Makdisi et Sadaka [35] et Makdisi et Marktanner [34].

${ }^{2}$ Appelée la guerre des Six Jours car, en six jours, l'armée israélienne remporte le conflit contre l'Egypte, la Jordanie et la Syrie.

${ }^{3}$ Il s'agit d'un traité inter-libanais, signé le 22 octobre 1989 à Taëf (Arabie-Saoudite), qui a imposé aux divers belligérants la fin de la guerre civile du Liban qui durait depuis 1975.
} 


\subsection{La configuration actuelle du parlement et la nouvelle réforme}

Le système actuel a été conçu pour assurer une bonne représentation des minorités, à la fois localement mais aussi au niveau national. Il s'agit notamment de préserver les intérêts des chrétiens, qui font la spécificité du Liban dans le Moyen-Orient à majorité musulmane. Les accords de Taëf ont permis un compromis sur le plan de la représentation politique. Ils ont fixé le nombre de députés à 128 , et désormais, le parlement ${ }^{4}$ compte 64 députés chrétiens et 64 musulmans. Ce souci d'équilibre marque aussi le rapport entre sunnites et chiites, dont la représentation est égale (27 députés) et proche de celle des maronites (34 députés). La configuration du parlement libanais est expliquée dans la deuxième colonne du tableau 1.

Les élections législatives libanaises, prévues en juin 2013, s'annonçaient houleuses, et ont été reportées. Le pays du Cèdre a adopté un nouveau système électoral. Le projet de loi électorale dit du "rassemblement orthodoxe" prévoit désormais, entre autres, la création de sièges supplémentaires au parlement : 3 sièges supplémentaires sont prévus pour les minorités chrétiennes et pour maintenir l'équilibre entre chrétiens et musulmans, trois autres sièges (un chiite, un sunnite et un druze) ont été ajoutés, portant le nombre total de sièges au parlement de 128 à 134. L'équilibre entre sunnites et chiites reste aussi assuré avec de nouveaux chiffres (28 sièges pour chaque groupe). La nouvelle configuration du prochain parlement ${ }^{5}$ est expliquée dans la troisième colonne du tableau 1.

TABLE 1 - Partage confessionnel des sièges au parlement libanais avant et après la réforme

\begin{tabular}{|l|c|c|}
\hline Confessions & Sièges avant réforme & Sièges après réforme \\
\hline Maronites & 34 & 34 \\
Grecs orthodoxes & 14 & 14 \\
Grecs catholiques & 8 & 8 \\
Arméniens orthodoxes & 5 & 5 \\
Arméniens catholiques & 1 & 1 \\
Evangélistes & 1 & 1 \\
Minorités chrétiennes & 1 & 4 \\
\hline Total confessions chrétiennes & 64 & 67 \\
\hline Sunnites & 27 & 28 \\
Chiites & 27 & 28 \\
Druzes & 8 & 9 \\
Alaouites & 2 & 2 \\
\hline Total confessions musulmanes & 64 & 67 \\
\hline \hline Total & 128 & 134 \\
\hline
\end{tabular}

Cette organisation du système électoral dans sa principale finalité de représentation des groupes confessionnels nous amène naturellement à le confronter aux réalités chiffrées de la population du Liban. Dans cette perspective, l'utilisation des listes électorales, considérées comme source officielle, est très instructive, même s'il convient d'en cerner les limites de validité. L'enjeu de l'article n'étant pas une présentation détaillée du système électoral libanais, nous retenons pour notre propos que, sur les listes électorales, la population est répartie entre 17 confessions et une catégorie autres confessions mais les autorités libanaises sont en mesure de classer la population selon la répartition confessionnelle au parlement, i.e. 11 groupes.

La population inscrite sur les listes électorales par confession pendant les principaux rendezvous d'élections législatives entre 2000 et $2014^{6}$ est donnée dans le tableau 2. Les chrétiens du

\footnotetext{
${ }^{4}$ Tous les quatre ans (en principe), les 128 députés de l'Assemblée libanaise - unique chambre législative du pays sont renouvelés.

${ }^{5}$ En raison des "tensions sécuritaires et politiques" qui s’intensifient dans la région, les élections ont été prévues dans un deuxième temps en 2014 mais le Parlement est prolongé jusqu'en 2017.

${ }^{6}$ Comme noté plus haut, l'élection a été prévue en 2013 (puis en 2014), cependant, le parlement est prolongé jusqu'en 2017.
} 
Liban représentent un peu moins de la moitié de la population de ce pays. Les estimations les plus courantes donnent un chiffre autour de $40 \%$ de chrétiens, soit le pourcentage le plus élevé de tous les pays du Proche et du Moyen-Orient. Néanmoins, ce taux ne cesse de baisser depuis plusieurs années. Le tableau 2 illustre très bien cette baisse de la population chrétienne dans l'ensemble de la population en tenant compte de la population enregistrée sur les listes électorales. Ainsi, ce taux variait autour de $44 \%$ en 2000 et oscille autour de $37 \%$ selon les données les plus récentes. Plusieurs éléments expliquent ce fait, notamment le taux de natalité relativement plus élevé chez les musulmans, essentiellement chez la population chiite, la forte émigration chrétienne, les naturalisations de population musulmane (réfugiés).

TABLE 2 - La population inscrite sur les listes électorales par confession entre 2000 et 2014 .

\begin{tabular}{|l|c|c|c|c|}
\hline Confession & 2000 & 2005 & 2009 & 2014 \\
\hline Maronites & 606553 & 666009 & 697553 & 696157 \\
Grecs orthodoxes & 226488 & 235702 & 242641 & 250094 \\
Grecs catholiques & 146644 & 156233 & 162519 & 166745 \\
Arméniens orthodoxes & 89649 & 90506 & 91241 & 89201 \\
Arméniens catholiques & 19392 & 20159 & 25611 & 19685 \\
Evangélistes & 18230 & 17357 & 12272 & 12789 \\
Minorités chrétiennes & 58819 & 41146 & 43419 & 75320 \\
\hline Total confessions chrétiennes & 1165775 & 1227112 & 1275256 & 1309991 \\
\hline Sunnites & 674571 & 792092 & 888875 & 970965 \\
Chiites & 638313 & 781767 & 873419 & 956275 \\
Druzes & 151971 & 168806 & 186496 & 193683 \\
Alaouites & 18491 & 23552 & 26918 & 27969 \\
\hline Total confessions musulmanes & 1483346 & 1766217 & 1975708 & 2148892 \\
\hline \hline Total Source : Ministère de l'intérieur libanais (en arabe)
\end{tabular}

Avant de traiter notre application des indices de pouvoir au parlement libanais, nous allons présenter nos premiers résultats, obtenus à partir d'un simple test de Khi2 entre la distribution (en \%) de la population de chaque confession et la répartition (en \%) du nombre de députés au parlement avant et après l'actuelle réforme. Le tableau 3 regroupe les pourcentages obtenus.

TABLE 3 - \% de la population et du nombre de sièges avant et après l'actuelle réforme.

\begin{tabular}{|l|c|c|c|c|c|c|}
\hline \multirow{2}{*}{ Confession } & \multicolumn{2}{|c|}{ \% sièges } & \multicolumn{4}{c|}{ \% population } \\
\cline { 2 - 7 } & avant réforme & après réforme & 2000 & 2005 & 2009 & 2014 \\
\hline Maronites & 26,56 & 25,37 & 22,90 & 22,25 & 21,46 & 20,13 \\
Grecs orthodoxes & 10,94 & 10,45 & 8,55 & 7,87 & 7,46 & 7,23 \\
Grecs catholiques & 6,25 & 5,97 & 5,54 & 5,22 & 5,00 & 4,82 \\
Arméniens orthodoxes & 3,91 & 3,73 & 3,38 & 3,02 & 2,81 & 2,58 \\
Arméniens catholiques & 0,78 & 0,75 & 0,73 & 0,67 & 0,79 & 0,57 \\
Evangélistes & 0,78 & 0,75 & 0,69 & 0,58 & 0,38 & 0,37 \\
Minorités chrétiennes & 0,78 & 2,99 & 2,22 & 1,37 & 1,34 & 2,18 \\
\hline Total confessions chrétiennes & 50 & 50 & 44,01 & 40,99 & 39,23 & 37,87 \\
\hline Sunnites & 21,09 & 20,90 & 25,46 & 26,46 & 27,34 & 28,07 \\
Chiites & 21,09 & 20,90 & 24,10 & 26,12 & 26,87 & 27,65 \\
Druzes & 6,25 & 6,72 & 5,74 & 5,64 & 5,74 & 5,60 \\
Alaouites & 1,56 & 1,49 & 0,70 & 0,79 & 0,83 & 0,81 \\
\hline Total confessions musulmanes & 50 & 50 & 55,99 & 59,01 & 60,77 & 62,13 \\
\hline \hline Total & 100 & 100 & 100 & 100 & 100 & 100 \\
\hline
\end{tabular}

Les tests du Khi2 nous amènent à rejeter l'hypothèse d'égalité des deux distributions testées (population et sièges), à la fois avant et après la réforme actuelle. Les résultats des tests sont indiqués dans le tableau 4. Notons que, concernant la nouvelle réforme, nous avons effectué ce 
test pour toutes les années alors que la réforme n'entre en application qu'à partir des prochaines élections. Le décalage entre le pourcentage de la population et celui des sièges peut aussi être vérifié au niveau de chaque grande confession : chrétienne et musulmane. Nous avons donc calculé le pourcentage de la population de chaque confession chrétienne par rapport à toute la population chrétienne ainsi que le pourcentage des sièges de chaque confession chrétienne par rapport au nombre de sièges accordés à la confession chrétienne dans l'ensemble. Ce calcul a été effectué avant et après la réforme et pour toutes les années. Par ailleurs, le même calcul a été effectué en prenant en compte l'autre grande confession musulmane. Au total, aucun des 16 tests du Khi2 effectués ne donne une similarité entre la distribution en pourcentage de la population et celle des sièges en pourcentage à l'intérieur de chaque grande confession.

Comme prévu, ces tests traduisent parfaitement l'absence de considération de la population dans le choix du nombre de sièges à affecter à chaque groupe confessionnel. En effet, soucieux de construire une nation tenant compte du pluralisme religieux, le système confessionnel est essentiellement basé sur des considérations politiques plutôt que sur le simple pourcentage de la population de chaque confession. Par conséquent, par rapport à leur poids en population, les nombres de sièges ont pour effet de sous-représenter la confession musulmane dans son ensemble et de sur-représenter la confession chrétienne. De même, à l'intérieur de chaque confession, le système a comme résultat de sous-représenter les grandes confessions et de sur-représenter les plus petites. Notons que la question de la répartition des sièges entre les agents (Etats, partis politiques, ...) et son lien avec la population a suscité un grand intérêt théorique et empirique. Une fois de plus, la littérature s'est considérablement accrue ces dernières années. Nous pouvons citer à titre d'exemple le livre de Balinski et Young [3] ou encore les travaux de Barthélémy et Martin [7, 8], Felsenthal et Machover [21, 23], Laruelle et Widgrén [31], Leech [32], Leech et Aziz [33], Turnovec [46], etc. Ces travaux montrent qu'il est difficile de construire une méthode fiable d'allocation des sièges permettant de mieux refléter les chiffres de la population.

TABLE 4 - Les tests du Khi2

\begin{tabular}{|l|c|c|c|c|}
\hline & 2000 & 2005 & 2009 & 2014 \\
\hline \hline $\begin{array}{l}\text { Variable test : sièges avant réforme en \%. } \\
\text { Les \% de la population représentent-ils une distribution } \\
\text { similaire aux \% des sièges de chaque confession? }\end{array}$ & Non & Non & Non & Non \\
\hline \hline $\begin{array}{l}\text { Variable test : sièges après réforme en \%. } \\
\text { Les \% de la population représentent-ils une distribution } \\
\text { similaire aux \% des sièges de chaque confession? }\end{array}$ & Non & Non & Non & Non \\
\hline
\end{tabular}

\section{La mesure du pouvoir dans le parlement libanais}

Les indices de pouvoir seront calculés pour deux types d'élections qui ont lieu au sein du parlement :

- L'élection présidentielle : c'est au Parlement qu'incombe la tâche de choisir le Président. Le vote doit être acquis aux $2 / 3$ des suffrages exprimés. Comme noté plus haut, la présidence libanaise revient à un chrétien maronite. Il est à noter que, même si, depuis la fin de la guerre civile, le chef de l'Etat ne dispose plus d'un véritable pouvoir exécutif comme autrefois, l'enjeu du scrutin a toujours transcendé la sphère politique nationale.

- La prise de décision classique : la majorité simple (50\% des voix plus une) constitue la règle de prise de décision classique au sein du parlement libanais pour voter les textes de loi. 


\subsection{Le cadre conceptuel des indices de pouvoir}

Afin de présenter notre application des indices de pouvoir, il est nécessaire d'avoir recours aux outils de la théorie des jeux coopératifs en considérant un jeu dans lequel les joueurs sont les groupes confessionnels. Cette théorie permet d'analyser la question de la répartition (pouvoir, profits, imputation de coûts, ...) dans une situation où les agents peuvent former des coalitions pour réaliser leurs objectifs. Nous nous appuierons sur les deux indices les plus connus, à savoir l'indice de Banzhaf [4] et celui de Shapley-Shubik [43]. Nous adoptons ces deux indices afin de rendre plus robustes les conclusions obtenues.

Pour notre application, le pouvoir est calculé dans le cadre d'un jeu de vote. Ce cadre est général à toute structure (assemblée, comité, ...) usant du vote pour prendre une décision. Un jeu de vote est définit par le couple $(N, W)$ où $N=\{1,2, \ldots, n\}$ est l'ensemble des joueurs et $W$ est l'ensemble des coalitions gagnantes, c'est-à-dire, celles ayant la capacité de remporter le vote. Dans cette configuration, l'abstention est exclue : un joueur n'a le choix de voter que oui ou non. Nous nous intéressons dans ce qui suit à un cas particulier de jeux de vote, à savoir les jeux de vote pondéré dans la mesure où ils sont particulièrement adaptés à notre application. Ce jeu est noté ici $\left(q ; w_{1}, \ldots, w_{n}\right)$. La notation $w_{i} \geq 0$ représente le poids de vote du joueur $i$ et $q$ est la règle de vote (le quota) utilisée : une coalition $S$ ne remporte une décision (i.e. $S \in W$ ) que lorsque la somme des poids des joueurs composant la coalition $S$ atteint au moins le quota $q$. Formellement,

$$
S \in W \Longleftrightarrow \sum_{i \in S} w_{i} \geq q
$$

Afin de présenter la formulation générale des deux indices, nous introduisons la notion de fonction caractéristique définit pour toute coalition $S \operatorname{par} v(S)$ :

$$
v(S)=1 \text { si } S \in W \quad \text { et } \quad v(S)=0 \text { si } S \notin W .
$$

En particulier, on attribue une valeur $v$ de 1 à chaque coalition gagnante $S$. Dans le cas contraire, la coalition est dite perdante et $v(S)=0$. Afin de mesurer le pouvoir de vote de chaque joueur, le critère suivant est utilisé : un joueur, ici un groupe confessionnel, exerce de l'influence dans un scrutin lorsqu'il est décisif (critique ou pivot). Un joueur est décisif s'il a la capacité à faire basculer une coalition perdante en coalition gagnante. Formellement, le joueur $i$ est décisif pour la coalition $S$ lorsque $v(S)=1$ et $v(S \backslash\{i\})=0$. La notation $S \backslash\{i\}$ étant la coalition $S$ moins le joueur $i$.

L'indice de pouvoir dû à Shapley et Shubik s'intéresse aux ordres des joueurs dans la formation des coalitions. L'indice prend en compte toutes les permutations possibles de formation de coalitions et le pouvoir de chaque joueur $i$, noté $\phi(i)$, est obtenu en divisant le nombre de permutations dans lesquelles le joueur $i$ est décisif par le nombre total de permutations possibles. Formellement,

$$
\phi(i)=\sum_{S \subseteq N} \frac{(s-1) !(n-s) !}{n !}[v(S)-v(S \backslash\{i\})] .
$$

$s$ étant le nombre de joueurs dans la coalition $S$.

Afin d'illustrer ces notions, prenons le jeu représenté par la structure suivante $(6 ; 4,3,2)$. Dans ce jeu, 6 voix au moins sont nécessaires pour qu'une proposition soit acceptée sachant que les poids des joueurs 1,2 et 3 sont respectivement 4,3 et 2 . Les six permutations possibles sont les suivantes : 123, 132, 213, 231, 312, 321. Dans chaque permutation, le joueur décisif figure en gras. Ainsi, le joueur 1 est décisif dans quatre permutations, alors que les joueurs 2 et 3 sont décisifs dans une seule permutation. Ceci définit la valeur de l'indice de pouvoir de Shapley-Shubik pour chaque joueur, soit $\phi(1)=\frac{4}{6}$ et $\phi(2)=\phi(3)=\frac{1}{6}$. 
L'indice de pouvoir de Banzhaf pour chaque joueur $i$, noté $\beta(i)$, est donné par la proportion des coalitions dans lesquelles $i$ est décisif, sur l'ensemble des occurrences où n'importe quel joueur est décisif. Formellement,

$$
\beta(i)=\frac{\sum_{S \subseteq N}[v(S)-v(S \backslash\{i\})]}{\sum_{j \in N} \sum_{S \subseteq N}[v(S)-v(S \backslash\{j\})]} .
$$

Dans notre exemple, les coalitions possibles sont $\{1\},\{2\},\{3\},\{1,2\},\{1,3\},\{2,3\}$ et $\{1,2,3\}$. Par ailleurs, les coalitions gagnantes sont $\{1,2\},\{1,3\}$ et $\{1,2,3\}$. Dans les coalitions $\{1,2\}$ et $\{1,3\}$, les deux joueurs sont décisifs. En revanche, dans la coalition $\{1,2,3\}$, seul le joueur 1 est décisif. En résumé, la proportion pour que le joueur 1 (respectivement 2 et 3 ) soit décisif est égale à $\frac{3}{5}$ (respectivement $\frac{1}{5}$ et $\frac{1}{5}$ ). Ceci définit la valeur de l'indice de pouvoir de Banzhaf pour chaque joueur, soit $\beta(1)=\frac{3}{5}$ et $\beta(2)=\beta(3)=\frac{1}{5}$.

\subsection{Analyse des résultats généraux}

Nous commençons cette section par analyser la capacité collective du parlement libanais à prendre des décisions au cours de chaque vote. En effet, nous avons calculé le pourcentage des coalitions gagnantes par rapport à toutes les coalitions possibles. Autrement dit, l'indicateur que nous utilisons dans cette partie mesure la capacité du système de vote à générer des coalitions gagnantes plutôt que des coalitions perdantes. Cet indicateur a été calculé, comme noté plus haut, pour les deux types de règles de vote. Nos résultats sont établis dans le tableau 5.

TABLE 5 - Pourcentage des coalitions gagnantes : système avant réforme comparé à celui après réforme pour la majorité simple et la majorité aux $2 / 3$.

\begin{tabular}{|l|l|c|c|}
\hline & Distribution & Majorité simple & Majorité aux $2 / 3$ \\
\hline \multirow{2}{*}{ Toutes confessions confondues } & avant réforme & $49,19 \%$ & $22,18 \%$ \\
\cline { 2 - 4 } & après réforme & $49,49 \%$ & $22,03 \%$ \\
\hline \multirow{2}{*}{$\begin{array}{l}\text { Coalitions n'incluant pas la grande } \\
\text { confession chrétienne (maronite) }\end{array}$} & avant réforme & $21,9 \%$ & $2,93 \%$ \\
\cline { 2 - 4 } & après réforme & $22,78 \%$ & $3,42 \%$ \\
\hline $\begin{array}{l}\text { Coalitions n'incluant pas les sunnites } \\
\text { ou les chiites }\end{array}$ & avant réforme & $28,45 \%$ & $8,21 \%$ \\
\cline { 2 - 4 } & après réforme & $28,15 \%$ & $7,82 \%$ \\
\hline $\begin{array}{l}\text { Coalitions n'incluant pas les sunnites } \\
\text { et les chiites }\end{array}$ & avant réforme & $7,63 \%$ & $0 \%$ \\
\cline { 2 - 4 } & après réforme & $6,85 \%$ & $0 \%$ \\
\hline
\end{tabular}

Le tableau 5 montre la difficulté inhérente au système confessionel libanais à former des coalitions gagnantes, tout particulièrement lorsqu'il s'agit du choix du président de la république. Ainsi, au niveau total, lorsqu'il s'agit de choisir le président, seuls $22,18 \%$ des coalitions sont gagnantes. La nouvelle réforme, où $22,03 \%$ des coalitions sont gagnantes, n'a presque aucun effet sur la capacité du parlement à choisir le président. De même, en excluant la grande confession maronite, le pourcentage des coalitions gagnantes oscille autour de $3 \%$ avant et après l'actuelle réforme. Par ailleurs, en excluant les sunnites ou les chiites, ce chiffre augmente et tourne autour de $8 \%$ avant et après la nouvelle réforme.

Ces chiffres montrent a priori la difficulté de l'élection présidentielle au Liban et mettent en lumière la complexité du système confessionnel et des jeux d'alliance qui font la politique de ce pays. Il ressort aussi que la mise en œuvre de la nouvelle réforme ne devrait rien changer dans la difficulté de la prise de décision collective pour le choix du président. De même, le pourcentage des coalitions gagnantes devient plus important lorsqu'un vote à la majorité simple est considéré. Ainsi, ce pourcentage oscille autour de $49 \%$ lorsque toutes les coalitions (toutes 
confessions confondues) sont considérées comme possibles. A l'image du cas de la majorité aux $2 / 3$, la nouvelle réforme a un effet sur le pourcentage des coalitions gagnantes, mais dans une proportion très limitée. De même, il y a lieu de remarquer que l'importance relative de l'influence des grandes coalitions par rapport aux petites coalitions samoindrit lorsque l'on considère un vote à la majorité simple au lieu de la majorité aux $2 / 3$. En effet, le pourcentage des coalitions gagnantes devient relativement plus important, rendant même possible une prise de décision avec des coalitions n'incluant ni les chiites ni les sunnites (7,63\% et 6,85\% des coalitions sont gagnantes avant et après la réforme, respectivement) lorsqu'une majorité simple est requise.

Nous avons ensuite calculé les indices de Banzhaf et Shapley-Shubik pour la pondération initiale ainsi que celle correspondante à la réforme. Les tableaux 6 et 7 illustrent ces résultats pour les deux types de majorités. A partir des calculs réalisés, plusieurs enseignements assez tranchés ressortent du tableau 6. Comme attendu, on note tout d'abord un décalage entre le pouvoir "réel" représenté par l'indice de Banzhaf ou l'indice de Shapley-Shubik et le pouvoir "apparent" donné par le pourcentage de sièges de chaque confession. A titre d'exemple, les sièges des maronites représentent $26,56 \%$ du total des sièges alors que l'indice de pouvoir de Banzhaf leur donne un pouvoir "réel" de $29,44 \%$ à la majorité simple et $26,62 \%$ à la majorité aux $2 / 3$. De même, le décalage entre les deux pouvoirs semble visible lorsque l'on considère les deux grandes confessions (chrétienne et musulmane). Au total, la constitution répartit à égalité le nombre de sièges entre musulmans et chrétiens, cependant, lensemble des pouvoirs "réels" à l'intérieur de chaque grande confession ne donne pas lieu à une parfaite égalité. Ainsi, lorsqu'une majorité simple est requise, le total du pouvoir de Banzhaf des confessions chrétiennes est égal à 47,66\% contre $52.34 \%$ pour les confessions musulmanes. Ce résultat est renforcé par l'indice de Shapley-Shubik et peut s'expliquer par l'éclatement des sièges entre trois confessions chrétiennes ayant chacune un siège (arméniens catholiques, évangélistes et minorités chrétiennes), rendant leur pouvoir très faible (uniquement 0,90\% avec l'indice de Banzhaf et 0,79\% avec celui de Shapley-Shubik).

TABLE 6 - Indices de Banzhaf et Shapley-Shubik avec la majorité simple et la majorité aux 2/3 pour le système avant réforme.

\begin{tabular}{|l|c|c|c|c|c|c|}
\hline \multirow{2}{*}{ Confession } & \multirow{2}{*}{ Sièges } & \multirow{2}{*}{$\%$ sièges } & \multicolumn{2}{|c|}{ Banzhaf en \% } & \multicolumn{2}{c|}{ Shapley-Shubik en \% } \\
\cline { 4 - 7 } & & & majorité simple & majorité aux 2/3 & majorité simple & majorité aux 2/3 \\
\hline Maronites & 34 & 26,56 & 29,44 & 26,62 & 29,40 & 27,78 \\
Grecs orthodoxes & 14 & 10,94 & 6,69 & 12,30 & 7,25 & 12,46 \\
Grecs catholiques & 8 & 6,25 & 5,42 & 6,76 & 5,63 & 6,59 \\
Arméniens orthodoxes & 5 & 3,91 & 3,42 & 3,11 & 4,04 & 2,70 \\
Arméniens catholiques & 1 & 0,78 & 0,90 & 1,08 & 0,79 & 1,11 \\
Evangélistes & 1 & 0,78 & 0,90 & 1,08 & 0,79 & 1,11 \\
Minorités chrétiennes & 1 & 0,78 & 0,90 & 1,08 & 0,79 & 1,11 \\
\hline Total confessions chrétiennes & 64 & 50 & 47,66 & 52,03 & 48,69 & 52,86 \\
\hline Sunnites & 27 & 21,09 & 22,38 & 19,32 & 21,86 & 19,09 \\
Chiites & 27 & 21,09 & 22,38 & 19,32 & 21,86 & 19,09 \\
Druzes & 8 & 6,25 & 5,42 & 6,76 & 5,63 & 6,59 \\
Alaouites & 2 & 1.56 & 2,16 & 2,57 & 1,98 & 2,38 \\
\hline Total confessions musulmanes & 64 & 50 & 52,34 & 47,97 & 51,31 & 47,14 \\
\hline \hline Total & 128 & 100 & 100 & 100 & 100 & 100 \\
\hline
\end{tabular}

Par ailleurs, il semble que les chrétiens dans l'ensemble deviennent gagnants sur le vote du président. En effet, lorsqu'une majorité aux $2 / 3$ est requise, le total du pouvoir de Banzhaf des confessions chrétiennes est égal à $52,03 \%$ contre $47,97 \%$ pour les confessions musulmanes. A nouveau, ce résultat est renforcé par l'indice de Shapley-Shubik et peut s'expliquer par le fait que le pouvoir des petites confessions devient relativement plus important que celui des grandes confessions dans le cas d'une majorité aux $2 / 3$. Autrement dit, le poids des plus gros (une 
confession chrétienne représentée par les maronites et deux confessions musulmanes représentées par les chiites et les sunnites) compte moins dans le processus de décision. Ainsi, même éclatées, les trois petites confessions chrétiennes citées ci-dessus bénéficieront d'un pouvoir accru dans l'ensemble (1,08\% contre $0,90 \%$ avec l'indice de Banzhaf et $1,11 \%$ contre $0,79 \%$ avec celui de Shapley-Shubik). Une autre raison est liée au pouvoir des grecs orthodoxes (14 sièges) : disposant de presque du double des sièges des grecs catholiques ( 8 sièges), le pouvoir de cette confession est presque égal à celui des grecs catholiques lorsqu'une majorité simple est requise alors que ce pouvoir augmente considérablement lorsqu'une majorité aux $2 / 3$ est demandée passant ainsi de $6,69 \%$ à 12,30\% avec l'indice de Banzhaf et de 7,25\% à 12,46\% avec celui de Shapley-Shubik.

Ces chiffres nous conduisent naturellement à une comparaison entre le pouvoir "réel" de chaque confession lorsque la majorité aux $2 / 3$ est requise au lieu de la majorité simple. Comme noté plus haut, il est clair que le poids des plus grandes confessions devient moins important dans le cas de la majorité aux 2/3. A titre d'exemple, le pouvoir des maronites passe de 29,44\% à 26,62\% avec l'indice de Banzhaf et de $29,40 \%$ à 27,78\% avec celui de Shapley-Shubik. Par ailleurs, le pouvoir "réel" s'améliore pour toutes les autres confessions, à l'exception des arméniens orthodoxes, avec un effet positif très net chez les grecs orthodoxes quel que soit l'indice de pouvoir utilisé.

Le tableau 7 illustre le pouvoir "apparent" donnée par le pourcentage des sièges dans la nouvelle configuration du parlement ainsi que le pouvoir "réel" de chaque confession pour les deux types de majorités. D'une manière générale, ajouter des sièges à quelques confessions tout en maintenant l'équilibre confessionnel entre chrétiens et musulmans modifie légèrement le pouvoir de chaque confession sans bouleverser les résultats obtenus pour la configuration à 128 sièges.

TABLE 7 - Indices de Banzhaf et Shapley-Shubik avec la majorité simple et la majorité aux 2/3 pour le système réformé.

\begin{tabular}{|c|c|c|c|c|c|c|}
\hline \multirow{2}{*}{ Confession } & \multirow{2}{*}{ Sièges } & \multirow{2}{*}{ \% sièges } & \multicolumn{2}{|c|}{ Banzhaf en \% } & \multicolumn{2}{|c|}{ Shapley-Shubik en \% } \\
\hline & & & majorité simple & majorité aux $2 / 3$ & majorité simple & majorité aux $2 / 3$ \\
\hline Grecs orthodoxes & 14 & 10,45 & 6,20 & 11,69 & 6,90 & 12,28 \\
\hline Arméniens orthodoxes & 5 & 3,73 & 3,47 & 4,07 & 3,72 & 3,67 \\
\hline Arméniens catholiques & 1 & 0,75 & 0,63 & 0,73 & 0,63 & 0,58 \\
\hline Evangélistes & 1 & 0,75 & 0,63 & 0,73 & 0,63 & 0,58 \\
\hline Total confessions chrétiennes & 67 & 50 & 47,42 & 52,17 & 48,44 & 52,69 \\
\hline Sunnites & 28 & 20,90 & 22,92 & 19,44 & 22,29 & 19,51 \\
\hline Chiites & 28 & 20,90 & 22,92 & 19,44 & 22,29 & 19,51 \\
\hline Druzes & 9 & 6,71 & 5,36 & 7,28 & 5,67 & 6,81 \\
\hline Alaouites & 2 & 1,49 & 1,37 & 1,67 & 1,30 & 1,49 \\
\hline Total confessions musulmanes & 67 & 50 & 52,58 & 47,83 & 51,56 & 47,31 \\
\hline
\end{tabular}

En effet, l'écart entre les deux types de pouvoirs est maintenu avec la nouvelle configuration pour les deux indices de pouvoir utilisés. A titre d'exemple, les sièges des maronites représentent $25,37 \%$ du total des sièges alors que l'indice de pouvoir de Banzhaf leur donne un pouvoir "réel" de $28,71 \%$ à la majorité simple et $25,45 \%$ à la majorité aux $2 / 3$. De même, au niveau du pouvoir "réel" de chaque grande confession (chrétienne ou musulmane) dans son ensemble, nos résultats montrent que le changement est marginal. En effet, le total de tous les pouvoirs "réels" de chaque grande confession ne donne pas une parfaite égalité entre les deux confessions chrétienne et musulmane. Ceci est vrai pour l'indice de Banzhaf et renforcé par celui de Shapley-Shubik. De plus, les deux indices montrent que, a priori, les chrétiens deviennent "gagnants" dans l'ensemble sur le vote du président. 
Nous avons aussi effectué une comparaison entre le pouvoir à la majorité aux $2 / 3$ et celui de la majorité simple pour les deux indices. En prenant en compte l'indice de Banzhaf, il s'avère que les confessions pour lesquelles on observe une baisse de pouvoir sont données uniquement par les grandes confessions, à savoir les chiites, les maronites et les sunnites. Pour certaines confessions, l'effet est nul ou quasi-nul comme c'est le cas pour les deux petites confessions. Toutefois, l'effet net des autres confessions est positif, avec un avantage nettement positif pour la deuxième plus grande confession chrétienne. Ces résultats sont globalement renforcés par l'indice de ShapleyShubik. En effet, contrairement à l'indice de Banzhaf, on observe que le pouvoir des arméniens orthodoxes, arméniens catholiques et des évangélistes baisse légèrement lorsque la majorité aux $2 / 3$ est requise au lieu de la majorité simple.

Des questions tout à fait légitimes peuvent être posées concernant la mise en place de la nouvelle réforme et son effet sur le pouvoir de chaque confession. Dans cette partie, nous souhaitons identifier, en terme de pouvoir "réel", les perdants et les gagnants de la réforme. Nos résultats nous permettent d'effectuer cette comparaison pour les deux règles de vote ainsi que les deux indices de pouvoir. En prenant en compte l'indice de Banzhaf et la majorité simple, la figure 1 illustre cette idée. A ce niveau, plusieurs paradoxes méritent d'être mentionnés. Ainsi, seuls trois confessions (minorités, chiites et sunnites) des quatre groupes qui ont gagné plus de sièges (minorités, chiites, sunnites et druzes) ont gagné en terme de pouvoir "réel". Le quatrième groupe, à savoir les druzes, malgré son gain d'un siège, a vu son pouvoir "réel" baisser légèrement. A l'inverse, les arméniens orthodoxes ont gardé le même nombre de sièges et ont vu leur pouvoir "réel" augmenter légèrement. Finalement, les autres groupes ont gardé le même nombre de sièges mais leur pouvoir "réel" a chuté de façon non négligeable. En prenant en compte l'indice de Shapley-Shubik et la majorité simple, il ressort que ces résultats sont relativement différents. En effet, toutes les confessions, dont le nombre de sièges n'est pas modifié, voient leur pouvoir de vote baisser. A l'inverse, toutes les confessions, qui ont obtenu des sièges supplémentaires grâce à la réforme, voient leur pouvoir de décision augmenter dans le cas des votes à la majorité simple.

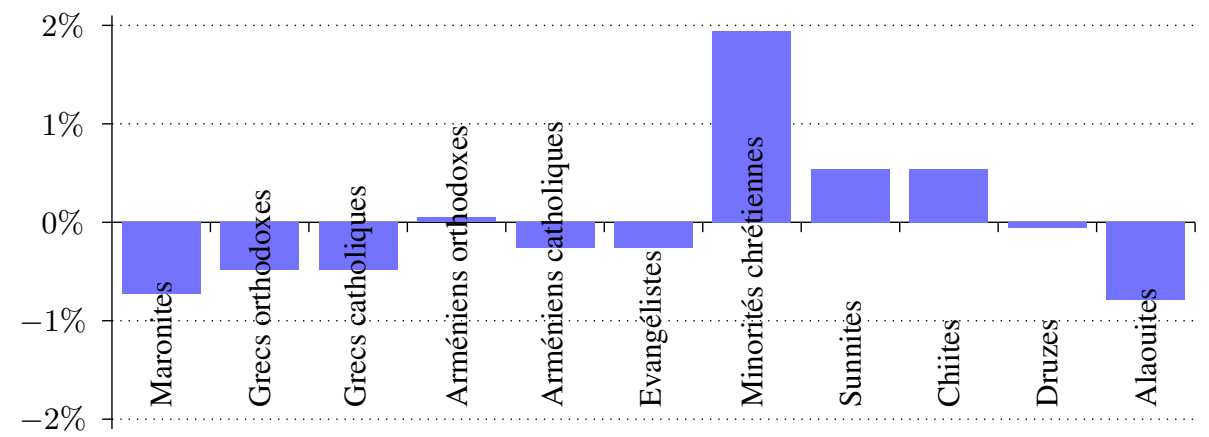

FIGURE 1 - Effets nets de la réforme sur le pouvoir des confessions avec la majorité simple : pouvoir de Banzhaf après réforme moins celui avant réforme.

Le même raisonnement est utilisé pour l'indice de Banzhaf et la majorité aux $2 / 3$ illustré dans la figure 2. A partir des calculs réalisés avec l'indice de Banzhaf pour la majorité aux $2 / 3$, il ressort que toutes les confessions qui ont gagné des sièges dans la nouvelle configuration ont vu leur pouvoir d'influence effectivement augmenter lorsqu'il s'agit d'un vote à la majorité aux $2 / 3$ par rapport à la configuration initiale. De même, on constate aussi que les arméniens orthodoxes sont aussi des gagnants de la nouvelle réforme. De la même manière, les autres groupes ont gardé le même nombre de sièges mais leur pouvoir "réel" a chuté de façon non négligeable. Ces remarques sont renforcées par les calculs effectués avec l'indice de Shapley-Shubik dans la mesure où nous 
avons pu remarquer les mêmes effets.

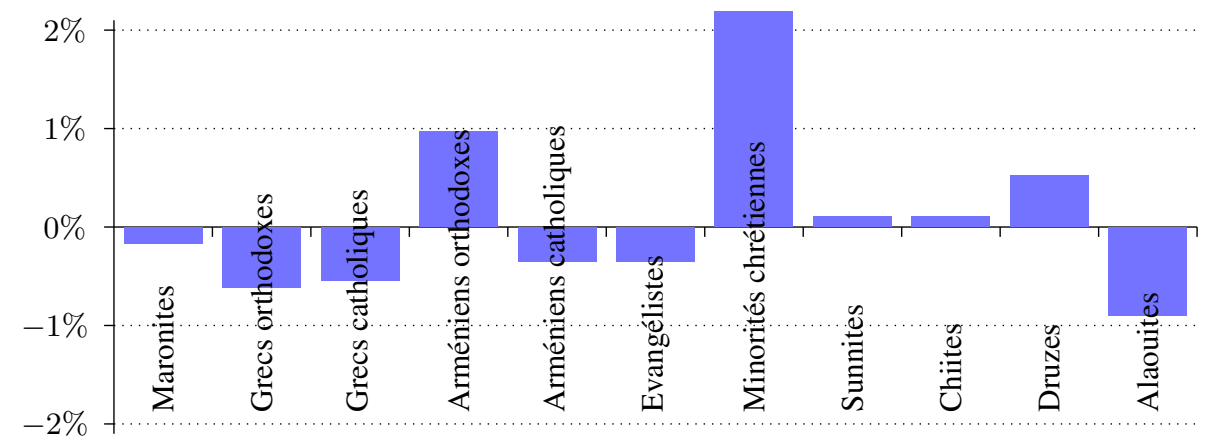

FIGURE 2 - Effets nets de la réforme sur le pouvoir des confessions avec la majorité aux $2 / 3$ : pouvoir de Banzhaf après réforme moins celui avant réforme.

\section{Conclusion}

Le système électoral libanais consistant à partager les sièges du Parlement entre les confessions présente des caractéristiques non apparentes mais importantes. En effet, il nous a paru central de faire cette première analyse à travers des éléments de la théorie des jeux coopératifs. Il nous semble que l'article propose une étude intéressante du pouvoir a priori de chaque confession pour l'ancienne et la nouvelle configuration prévue pendant les prochaines élections législatives. Cette nouvelle réforme suscite bien des critiques et des inquiétudes dans un pays marqué par une situation sécuritaire fortement instable. Nous avons utilisé des moyens mathématiques standards, comme l'indice de pouvoir de Banzhaf et celui de Shapley-Shubik, afin de mesurer le pouvoir "réel" et étudier l'effet de la nouvelle réforme sur le pouvoir de chaque confession.

Les premiers résultats que nous obtenons sont intéressants notamment parce qu'ils mettent à jour certains paradoxes. L'indice de Banzhaf nous apprend par exemple que les druzes, qui ont obtenu un siège supplémentaire suite à la réforme, voient leur pouvoir de décision diminuer dans les votes à la majorité simple. Par ailleurs, les arméniens orthodoxes, dont le nombre de sièges n'est pas modifié, voient leur pouvoir de vote augmenter. Nous avons aussi montré que la nouvelle réforme n'est pas susceptible d'exercer un impact notable sur la structure des pouvoirs des confessions. Dans cette perspective, les nouvelles conclusions ne sont pas très différentes de celles de l'ancienne configuration.

Il est évident que certaines hypothèses impliquent de prendre avec précaution les résultats de notre article. Ainsi, nous supposons que les membres d'une confession votent de la même façon. Nous analysons le pouvoir de chaque confession tout en sachant que les membres peuvent avoir des opinions différents sur le sujet du vote. Une telle hypothèse nous empêche de traiter des divisions internes à une communauté, en particulier les maronites qui affaiblissent d'un point de vue électoral leur poids au parlement. Par ailleurs, nous supposons que les confessions votent de manière indépendante les unes des autres. Il serait intéressant de voir les circonstances pour lesquelles cette hypothèse pourrait être abandonnée. En effet, nous espérons poursuivre notre analyse par une étude de la formation des coalitions sur le terrain. Le cas des druzes constitue un exemple révélateur : ceux-ci, étant donné leur coalition persistante avec les sunnites voient leur capacité (de facto) de négociation s'améliorer malgré la faiblesse de leur pouvoir réel attestée par les deux indices dans notre étude. D'un point de vue théorique les travaux d'Owen $[37,40]$ sur les jeux avec structure de coalitions nous seraient d'une grande aide afin d'étudier en détail la formation des coalitions dans le parlement libanais. 


\section{Références}

[1] J.M. Alonso-Meijide, B. Casas-Méndez, and M.G. Fiestras-Janeiro. Computing BanzhafColeman and Shapley-Shubik power indices with incompatible players. Applied Mathematics and Computation, 252 :377-387, 2015.

[2] N. Andjiga, F. Chantreuil, and D. Lepelley. La mesure du pouvoir de vote. Mathématiques et Sciences Humaines, 163 :111-145, 2003.

[3] M.L. Balinski and P. Young. Fair Representation : Meeting the Ideal of One Man, One Vote. New Haven and London, 1982.

[4] J. F. Banzhaf. Weighted voting doesn't work: A mathematical analysis. Rutgers Law Review, 19(2) :317-343, 1965.

[5] F. Barthélémy, A. Béraud, and M. Martin. Loi relative aux libertés et responsabilités des universités (loi LRU), élection du président et conseil d'administration : une analyse en termes de pouvoir. Revue d'économie politique, $3: 299-315,2008$.

[6] F. Barthélémy, A. Béraud, and M. Martin. La loi LRU a-t-elle modifié les distributions de pouvoir au sein des universités françaises. Revue économique, 60 :1469-1481, 2009.

[7] F. Barthélémy and M. Martin. Critères pour une meilleure répartition des sièges au sein des structures intercommunales. Une application au cas du Val-d'Oise. Revue économique, $58: 399-425,2007$.

[8] F. Barthélémy and M. Martin. A comparison between the methods of apportionment using power indices. The case of the US presidential election. Annales d'Economie et de Statistique, 101/102 :87-106, 2011.

[9] R. Barua, S.R. Chakravarty, and S. Roy. A new characterization of the Banzhaf index of power. International Game Theory Review, 7 :545-553, 2005.

[10] P. Berthelot. Vers une polarisation durable de la vie politique libanaise ? Maghreb-Machrek, $205: 7-30,2010$.

[11] F. Bisson, J. Bonnet, and D. Lepelley. La détermination du nombre de délégués au sein des structures intercommunales : une application de l'indice de pouvoir de Banzhaf. Revue d'Economie Régionale et Urbaine, 2 :259-282, 2004.

[12] F. Bobay. La réforme du conseil de l'union européenne à partir de la théorie des jeux. Revue Française d'Economie, 16(2) :3-61, 2001.

[13] F. Bobay. Constitution européenne : redistribution du pouvoir des Etats au conseil de l'UE. Economie et Prévision, 163(2) :101-115, 2004.

[14] J.S. Coleman. Control of Collectivities and the Power of a Collectivity to Act. In B. Lieberman (ed), Social Choice, New York, Gordon and Breach, pp 269-300, 1971.

[15] J. Colomer. Measuring parliamentary deviation. European Journal of Political Research, $30: 87-101,1996$.

[16] J. Colomer and F. Martinez. The paradox of coalition trading. Journal of Theoretical Politics, $7: 41-63,1995$. 
[17] I.J. Curiel. A class of non-normalized power indices for simple games. Mathematical Social Sciences, $13: 141-152,1987$.

[18] J. Deegan and E.W. Packel. A new index of power for simple n-person games. International Journal of Game Theory, 7 :113-123, 1978.

[19] P. Dubey. On the uniqueness of the Shapley value. International Journal of Game Theory, $4: 131-139,1975$.

[20] P. Dubey and L.S. Shapley. Mathematical properties of the Banzhaf power index. Mathematics of Operations Research, 4 :99-131, 1979.

[21] D.S. Felsenthal and M. Machover. The Measurement of Voting Power, Theory and Practice. Cheltenham, Edward Elgar, 1998.

[22] D.S. Felsenthal and M. Machover. The treaty of Nice and qualified majority voting. Social Choice and Welfare, 18 :431-464, 2001.

[23] D.S. Felsenthal and M. Machover. Analysis of QM rules in the draft constitution for Europe proposed by the European Convention. Social Choice and Welfare, 23 :1-20, 2003.

[24] G. Garrett and G. Tsebelis. Why resist the temptation to apply power indices to European Union? Journal of Theoretical Politics, 11 :291-308, 1999.

[25] K. Herne and H. Nurmi. The distribution of a priori voting power in the EC council of ministers and the european parliament. Scandinavian Political Studies, 16(3) :269-284, 1993.

[26] M. Holler and H. Nurmi. Power, Voting, and Voting Power : 30 Years After. Springer, Verlag, 2013.

[27] M. Holler and E. Packel. Power, luck and the right index. Journal of Economics, 43 :21-29, 1983.

[28] R.J. Johnston. On the measurement of power : some reaction to laver. Environment and Planning, 10 :907-914, 1978.

[29] H. Kauppi and M. Widgrén. Voting rules and budget allocation in an enlarged EU. European Journal of Political Economy, 23 :693-706, 2007.

[30] A. Laruelle and F. Valenciano. Shapley-Shubik and Banzhaf indices revisited. Mathematics of Operations Research, $26: 89-104,2001$.

[31] A. Laruelle and M. Widgrén. Is the allocation of voting power among the EU states fair? Public Choice, 94 :317-339, 1998.

[32] D. Leech. Designing the voting system for the council of the European Union. Public Choice, $113: 437-464,2002$.

[33] D. Leech and H. Aziz. The Double Majority Voting Rule of the EU Reform Treaty as a Democratic Ideal for an Enlarging Union : an Appraisal Using Voting Power Analysis. In : Cichocki, M.A. and Zyczkowski, K. (eds) : Institutional Design and Voting Power in the European Union, 2013.

[34] M. Makdisi, S. Marktanner. Trapped by consociationalism : The case of Lebanon. Lecture and Working Paper Series No. 1, American University of Beirut : Institute of Financial Economics, 2008. 
[35] S. Makdisi and R. Sadaka. The lebanese civil war. Lecture and Working Paper Series, Institute of Financial Economics : American University of Beirut, pages 1-53, 2003.

[36] A.S. Nowak and T. Tradzik. An alternative characterization of the weighted Banzhaf value. International Journal of Game Theory, 29 :127-132, 2000.

[37] G. Owen. Values of games with a priori unions. In Henn, R. and Moeschlin, O. (eds.), Mathematical Economics and Game Theory. Springer Verlag, 1977.

[38] G. Owen. Characterization of the Banzhaf-Coleman index. SIAM Journal of Applied Mathematics, 35 :315-327, 1978.

[39] G. Owen. A Note on the Banzhaf-Coleman axioms. In : P. Ordeshook, ed., Game theory and political science (New York University Press, New York), 451-461, 1978.

[40] G. Owen. Modification of the Banzhaf-Coleman index for games with a priori unions. In : Holler M. (ed) Power, voting and voting power. Physica-Verlag, Wurzburg, pp 232-238, 1981.

[41] R. Owen. The political economy of Arab presidents for life - and after. Middle East Development Journal, 5(1) :1-13, 2013.

[42] L. Penrose. The elementary statistics of majority voting. Journal of the Royal Statistical Society, 109(1):53-57, 1946

[43] L.S. Shapley and M. Shubik. A method for evaluating the distribution of power in a committee system. American Political Science Review, 48 :787-792, 1954.

[44] P. Straffin. Homogeneity, independence and power indices. Public Choice, 30 :107-118, 1977.

[45] P. Straffin. Power Indices in Politics. In : S.J. Brams, W.F. Lucas and P.D. Straffin, Jr. (eds) : Political and Related Models, 256-321, Springer Verlag, New York, 1982.

[46] F. Turnovec. National, political and institutional influence in European Union decision making. Czech Economic Review, 2 :154-173, 2008. 